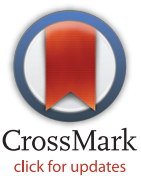

click for updates

\section{G OPENACCESS}

Citation: Park JK, Yang W, Katsnelson J, Lavker RM, Peng H (2015) MicroRNAs Enhance Keratinocyte Proliferative Capacity in a Stem CellEnriched Epithelium. PLoS ONE 10(8): e0134853. doi:10.1371/journal.pone. 0134853

Editor: Che John Connon, University of Reading, UNITED KINGDOM

Received: April 27, 2015

Accepted: July 14, 2015

Published: August 6, 2015

Copyright: @ 2015 Park et al. This is an open access article distributed under the terms of the Creative Commons Attribution License, which permits unrestricted use, distribution, and reproduction in any medium, provided the original author and source are credited.

Data Availability Statement: All relevant data are within the paper and its Supporting Information files.

Funding: The NU-SDRC is supported by the National Institute of Arthritis and Musculoskeletal and Skin Diseases (http://www.niams.nih.gov/) Grant AR057216. This research is supported by National Institutes of Health (https://www.nei.nih.gov/) Grants EY06769, EY017536 and EY019463 (to R.M.L.); a Dermatology Foundation (http:// dermatologyfoundation.org/) research grant and Career Development Award (to H.P.); and a MidWest Eye-Banks (http://www.eversightvision.org/) research grant (H.P.). The funders had no role in study design,
RESEARCH ARTICLE

\section{MicroRNAs Enhance Keratinocyte Proliferative Capacity in a Stem Cell-Enriched Epithelium}

\author{
Jong Kook Park ${ }^{1}$, Wending Yang ${ }^{1}$, Julia Katsnelson ${ }^{2}$, Robert M. Lavker ${ }^{1}$, Han Peng ${ }^{1}$ * \\ 1 Department of Dermatology, Northwestern University, Chicago, Illinois, United States of America, 2 Rush \\ University Medical Center, Chicago, Illinois, United States of America \\ * han-peng@northwestern.edu
}

\section{Abstract}

MicroRNAs are critical regulators of stem cell behavior. The miR-103/107 family is preferentially expressed in the stem cell-enriched corneal limbal epithelium and plays an important role in coordinating several intrinsic characteristics of limbal epithelial stem cells. To elucidate further the mechanisms by which miRs-103/107 function in regulating limbal epithelial stem cells, we investigate the global effects of miRs-103/107 on gene expression in an unbiased manner. Using antagomirs-103/107, we knocked down endogenous miRs-103/107 in keratinocytes and conducted an mRNA profiling study. We show that miRs-103/107 target mitogen-activated protein kinase kinase kinase 7 (MAP3K7) and thereby negatively regulate the p38/AP-1 pathway, which directs epithelial cells towards a differentiated state. Pharmacological inhibition of p38 increases holoclone colony formation, a measure of proliferative capacity. This suggests that the negative regulation of p38 by miRs-103/107 contributes to enhanced proliferative capacity, which is a hallmark of stem cells. Since miRs-103/107 also promote increased holoclone colony formation by regulating JNK activation through non-canonical Wnt signaling, we believe that this microRNA family preserves "stemness" by mediating the crosstalk between the Wnt/JNK and MAP3K7/p38/AP-1 pathways.

\section{Introduction}

Stem cells are a population of relatively undifferentiated cells with the capability to self-renew and give rise to progeny (transit amplifying; TA cells). Such progeny can also proliferate but their capacity is finite and once exhausted, these TA cells differentiate into specialized cell types $[1,2]$. Because of their high (infinite) proliferative capacity, stem cells play critical roles in tissue homeostasis and wound healing [3].

Cornea is comprised of three layers: epithelium, stroma, and endothelium. Like the epidermis, corneal epithelium functions as a dynamic barrier preventing entry of deleterious agents. Due to this protective function, the corneal epithelium is constantly shedding superficial cells, which must be replaced. Such a steady-state condition is, by definition, governed by stem cells, 
data collection and analysis, decision to publish, or preparation of the manuscript.

Competing Interests: The authors have declared that no competing interests exist. which are located in the basal layer of the limbal epithelium [4, 5], the transitional zone between cornea and conjunctiva. Limbal epithelial stem cells (LESCs) generate TA cells that migrate into the corneal epithelial basal layer [6-10]. These TA cells differentiate and migrate to the upper layers to replace the superficial cells that are continuously shed from the corneal epithelium during blinking. This steady-state process is critical for maintaining corneal epithelial homeostasis, and loss of LESCs because of eye diseases (e.g., ocular pemphigoid, StevensJohnson syndrome) or severe trauma (e.g., thermal and chemical burns) leads to corneal vascularization and opacification with severe visual loss $[11,12]$. Therefore, it is vital and clinically significant to understand the behavior of LESCs and identify factors that regulate LESC physiology.

microRNAs represent a major class of regulatory noncoding small RNAs that negatively control their target gene expression via inhibition of translation or degradation of mRNAs. microRNAs have emerged as important regulators of stem cell potency, proliferation, differentiation, and survival [13-24]. For example, miR-205 is critical for regulation of epithelial stem cells $[17,19]$. It controls stem cell proliferation and survival [19, 22], via targeting multiple negative regulators of the PI3K/Akt pathway including Frk, Inpp4b, Phlda3 and SHIP2 [19, 22]. $\mathrm{miR}-125 \mathrm{~b}$ is a positive regulator of stem cell expansion and required for preserving a healthy stem cell pool by targeting Vdr, Trp53lnp1, Scarb1, and FGFR2 [20, 23]. In contrast, miR-203 has been suggested as a suppressor of epidermal stem cells [14, 15, 21]. miR-203 functions in promoting and maintaining epidermal stem cell differentiation through inhibition of its targets p63, Skp2 and Msi2 [14, 15, 21]. Thus microRNAs can regulate different characteristics of stem cells in the epidermis and hair follicle epithelium by modulating various downstream signaling pathways. Another well-studied stem cell-TA cell system is the limbal/corneal epithelium [4-10, 24-27]. Surprisingly, our understanding of how limbal epithelial stem cells are regulated by microRNAs is limited. We have begun to address this knowledge gap by isolating relatively pure populations of limbal basal (stem cells) and corneal basal (TA cells) epithelial cells using laser capture microdissection. Following microRNA expression profiling, we identified nine microRNAs that are preferentially expressed in the stem cell-enriched limbal basal epithelium [28]. Among them, we demonstrated that microRNAs-103/107(miRs-103/107) promote a slow cycling phenotype, enhance proliferative capacity, and maintain proper cellcell communication in limbal epithelial stem cells [28].

In an effort to understand better how microRNAs affect limbal epithelial stem cell function, we employ gene function clustering analysis to connect the downstream target genes of limbalpreferred microRNAs to functional ontological pathways [28]. This unbiased analysis suggests that diverse processes are regulated by these limbal preferred microRNAs. Using bioinformatic analysis and biochemistry, we demonstrate that p38/AP-1 is a key downstream pathway of miRs-103/107 that contributes to the maintenance of stem cells.

\section{Materials and Methods Mice}

All animals were obtained from the Charles River Laboratories. Whole eye globes were collected from wild-type female mice $(\mathrm{Balb} / \mathrm{c})$ immediately after sacrifice and rapidly embedded in OCT compound and stored at $-80^{\circ} \mathrm{C}$.

\section{Ethics Statement}

All the procedures involving animals were approved by the Northwestern University Animal Care and Use Committee (NUACUC). 


\section{Laser capture microdissection and microRNA qPCR}

A PALM laser capture system (LCM; Zeiss) was used to isolate basal cells from limbal epithelium as previously described [28-30]. Taking advantage of the sharp demarcation between limbal and corneal epithelia based on the distinct morphological differences, $\sim 40$ cells/per limbus were captured avoiding the limbal/corneal junction as performed routinely by the laboratory [28-30]. Total cellular RNA from LCM-captured cells from individual mouse was isolated and purified using Qiagen miRNeasy kit (Qiagen). MicroRNA levels were measured using Exiqon's miRCURY LNA Universal RT miRNA PCR following the manufacturer's instructions.

\section{Known targets of microRNAs and Gene Ontology analysis}

Known target gene lists of microRNAs were exported from miRTarBase, which is a database for experimentally validated microRNA-target interactions. Functional Annotation Clustering of known target genes by specific microRNAs was performed in DAVID Functional Annotation Bioinformatics Resources v6.7.

\section{Cell culture and antagomir treatment}

To isolate and culture primary human limbal epithelial keratinocytes (HLEKs), cadaver donor corneas obtained from Eversight (Ann Arbor, MI) were explanted in CnT-20 media with supplements (CellnTech) [28]. For antagomir treatment, keratinocytes were incubated with $1 \mu \mathrm{M}$ antagomir, which can dramatically diminish the endogenous targeted microRNA $[2,28]$. Antagomirs sequences are $5^{\prime}-\mathrm{mU}\left({ }^{*}\right) \mathrm{mC}\left({ }^{*}\right) \mathrm{mA}\left({ }^{*}\right) \mathrm{mUmAmGmCmCmCmUmGmUmAmCmAmA}$ mUmGmCmU $\left({ }^{*}\right) \mathrm{mG}\left({ }^{*}\right) \mathrm{mC}\left({ }^{*}\right) \mathrm{mU}-\mathrm{Chol}-3^{\prime}$ (antagomir-103), 5' $-\mathrm{mU}\left({ }^{*}\right) \mathrm{mG}\left({ }^{*}\right) \mathrm{mA}\left({ }^{*}\right)$ mUmAmGmCmCmCmUmGmUmAmCmAmAmUmGmCmU $\left({ }^{*}\right) \mathrm{mG}\left({ }^{*}\right) \mathrm{mC}\left({ }^{*}\right) \mathrm{mU}-\mathrm{Chol}-3^{\prime}$ (antagomir-107), $5^{\prime}-m G\left({ }^{*}\right) m G\left({ }^{*}\right) m C\left({ }^{*}\right) m A m U m U m C m A m C m C m G m C m G m U m G m C m C ~\left({ }^{*}\right) m U\left({ }^{*}\right)$ $\mathrm{mU}\left({ }^{*}\right) \mathrm{mA}-\mathrm{Chol}-3^{\prime}$ (irrelevant-antagomir; antago-124). Antago-124 was used as an irrelevant control since miR-124 is a neuronal specific microRNA [31] and undetectable in human limbal epithelium [24]. Consistently, antago-124 treatment in human limbal/corneal epithelia was not distinguishable biochemically and physiologically compared with untreated controls $[22,32]$. "mN": 2'-Omethyl-modified oligonucleotide. “(*)": phosphorothioate linkage. "Chol”: cholesterol. All antagomirs were obtained from Dharmacon (Lafayette, CO).

\section{mRNA expression profiling and real time qPCR}

HLEKs were treated with antagomir-103,-107 and irrelevant-antagomir and subsequently total cellular RNAs were isolated and purified by an mRNeasy kit (Qiagen, Hilden, Germany). Agilent Bioanalyser 2100 (Agilent, Santa Clara, CA, USA) was used to determine RNA quality. Samples with RIN >9 were used for the following experiment. Expression profiling was conducted using an Illumina chip (Illumina HumanHT-12 kit[28]). Differentially expressed genes were imported into DAVID Functional Annotation Bioinformatics Resources v6.7 and analyzed by Functional Annotation Clustering. To confirm the results of microarray profiling, cDNA was prepared using SuperScript II reverse transcription kit (Invitrogen). Real-time qPCR was performed on a Roche LightCycler 96 System using the Roche FastStart Essential DNA Green Master (Roche) according to the manufacturer's instructions. Primer sequences used in this study were designed by Integrated DNA Technologies (IDT, Skokie, IL; S1 Table).

\section{Network Building}

GeneGo program was used to build a network using the MetaCore Database and the Direct Interactions algorithm. This algorithm creates a network consisting only of the seed nodes and 
of their direct interactions; no other objects from the database are added. Seed nodes included: (i) the differentially expressed genes in antagomir-103/107-treated HLEKs (S2 and S3 Tables) and (ii) stem-cell-maintenance-related direct target genes of miRs-103/107 (S4 Table).

\section{Western Blotting}

Western blots were performed as described previously [29]. The following antibodies were used at 1:1000 dilution: rabbit monoclonal anti-p-p38 (\#4511, Cell Signaling Technologies), rabbit monoclonal anti-MAP3K7(\#5206, Cell Signaling Technologies), rabbit monoclonal anti-p-MAP3K7(\#9339, Cell Signaling Technologies), rabbit monoclonal anti-p-c-Jun(\#2361, Cell Signaling Technologies), rabbit monoclonal anti-c-Jun(\#9165, Cell Signaling Technologies), rabbit monoclonal anti-p38(\#8699, Cell Signaling Technologies), and rabbit polyclonal anti-GAPDH (sc-25778, Santa Cruz Biotechnology).

\section{Luciferase reporter assay}

The untranslated region (3'UTR) of MAP3K7 was ligated into the psiCHECK-2 vector (Promega) and luciferase reporter assay was conducted as described in detail previously [28]. Primer sequences for MAP3K7 3'UTR: sense, CTGCCTCGAGATTCTCTGGGACCGTTACAT; antisense, ATTAGCGGCCGCCAAATACATGAGAAAACAATCCAAGAATCA.

\section{Colony Formation assay}

As previously described [28], HLEKs (200 cells per $100 \mathrm{~mm}$ dish) were seeded and incubated in FAD medium (DMEM/F12 media, FCS (10\%), insulin ( $5 \mu \mathrm{g} / \mathrm{ml})$, adenine $(0.18 \mathrm{mM})$, hydrocortisone $(0.4 \mu \mathrm{g} / \mathrm{ml})$, cholera toxin $(10 \mathrm{ng} / \mathrm{ml})$, triiodothyronine $(5 \mu \mathrm{g} / \mathrm{ml}), 5 \mu \mathrm{g} / \mathrm{ml}$ Human apotransferrin, glutamine $(4 \mathrm{mM})$, penicillin-streptomycin $(50 \mathrm{IU} / \mathrm{ml})$, and Epidermal growth factor $(10 \mathrm{ng} / \mathrm{ml}))$ with mitomycin C-treated 3T3 feeder cells. To test whether inhibition of p38 can promote colony formation, HLEKs were pre-treated with p38 inhibitor SB203580 $(10 \mu \mathrm{M})$ or DMSO for 3 days and then plated in FAD medium. After 2 weeks, the cells were fixed and stained with methanol/crystal violet. Image J program was used to measure colonies.

\section{Statistical analysis}

The significance of the differences between 2 groups was evaluated by an unpaired Student's $t$ test. The significance of the differences between 3 groups was tested by non-parametric oneway ANOVA.

\section{Results}

Previously, we employed a high throughput microRNA profiling analysis to identify limbalpreferred microRNAs [28]. Among hundreds of microRNAs, we identified nine that were highly expressed in the stem cell-enriched limbal epithelial basal cells compared with TA cellenriched central corneal epithelial basal cells [28]. To characterize their expression during mouse corneal development, we conducted real time qPCR for these nine microRNAs. RNAs were isolated from LCM-captured unperturbed limbal epithelial basal cells from the eyes of postnatal mice at day 3, 7, 14 and 60 . Such time points represent different and critical developmental stages of limbal epithelium in post-natal life. For example (i) day 3 -K14 appears primarily in the basal cells $[33,34]$; (ii) day 7 -there is a marked increase in corneal epithelial proliferation [35]; (iii) day 14 -is time of eye opening; and; (iv) day 60 -reflects a mature limbal epithelium [36]. We examined the expression of miR-184 and miR-31, which are two highly corneal-preferred microRNAs [22, 29, 37]. Consistently, levels of miR-184 and miR-31 were 


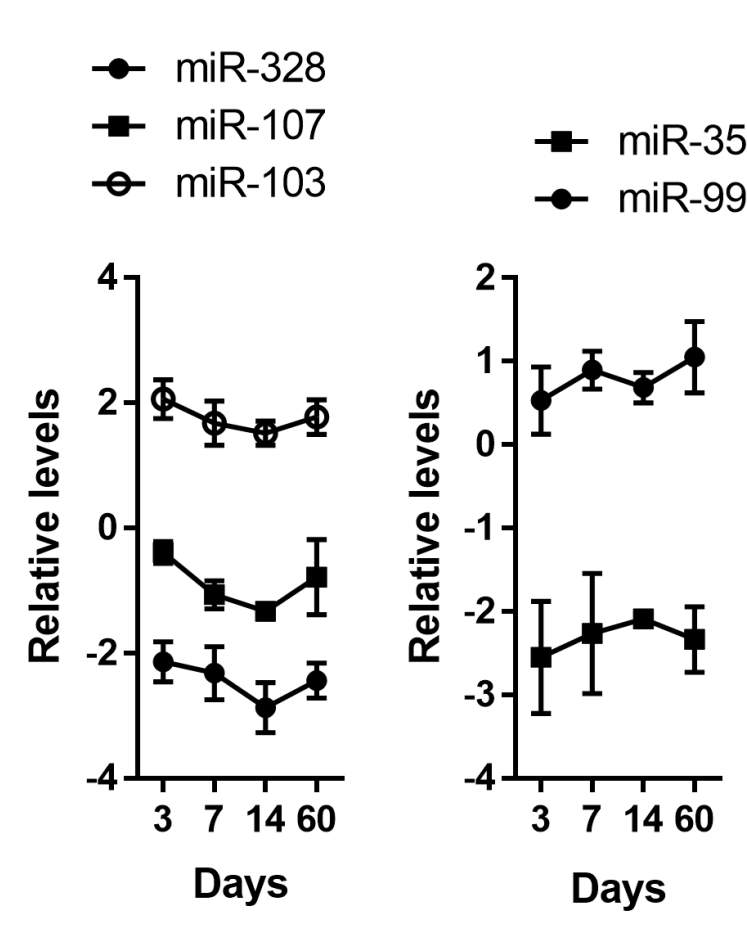

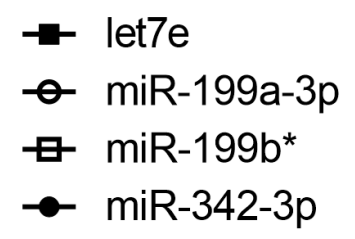

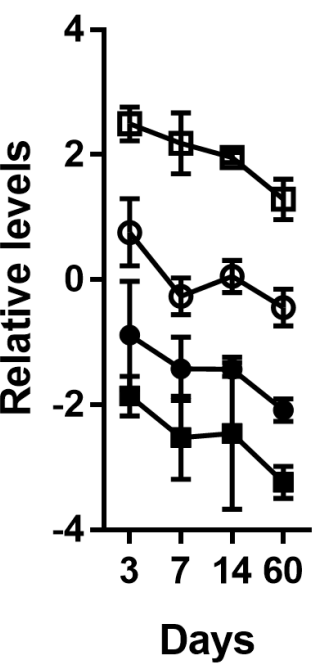

Fig 1. Expressions of limbal preferred microRNAs during postnatal eye development. MicroRNA qPCR analysis of miR-328, miR-103, miR-107, miR-350, miR-99a, let7e, miR-199a-3p, miR-199b*, and miR$342-3 p$ levels in limbal epithelium at postnatal day $3,7,14$, and 60 . Values are means \pm SD of three independent experiments.

doi:10.1371/journal.pone.0134853.g001

lower in the RNAs from isolated limbal epithelium compared with the RNAs from corneal epithelium (S1 Fig), confirming the relative purity of the isolated limbal epithelial cells. Interestingly, examination of the temporal expression of the nine miRNAs, revealed three types of patterns (Fig 1). Expression of let7e, miR-199a-3p, miR-199b*, and miR-342-3p decreased over time, whereas expression of miR-350 and miR-99a was relatively constant over the time periods analyzed. A third, more complex expression pattern was observed for miR-328, miR103 and miR-107. Expression of these miRNAs decreased between day 3 and day 14 and then increased by day 60 (Fig 1). Such a "V" shape expression pattern implies a more dynamic regulation of miR-328, miR-103 and miR-107 indicative of multiple functions during corneal development.

It's not surprising that one microRNA can regulate multiple processes since a microRNA can target hundreds of genes [38-40]. With respect to limbal epithelial basal cells, we have demonstrated that miRs-103/107 target p90RSK2, Wnt3a, Nedd9 and PPTPRM and thus play roles in maintaining slow cycling, high proliferative capacity, and proper cell-cell communication [28], which are well-accepted characteristics of epithelial stem cells. Interestingly, our unbiased Functional Annotation Clustering analysis also indicated that miRs-103/107 functioned in the process of "stem cell maintenance" (Fig 2).

miRs-103/107, which belong to a microRNA family, have identical sequences except for one nucleotide at the 3 '-end and regulate overlapping targets [41]. Thus, to determine which processes were regulated by miRs-103/107, we examined the genes commonly regulated by this microRNA family [28]. We found that 123 genes were significantly changed following knock down of miRs-103/107 by antagomir treatment (adjusted p-value (false discovery rate) $<0.05$ and fold change $>1.4 ; \mathrm{S} 2$ and S3 Tables). Functional Annotation Clustering analysis revealed 


\title{
Enrichment Scores
}

\author{
$\begin{array}{llllllll}0 & 0.5 & 1 & 1.5 & 2 & 2.5 & 3 & 3.5\end{array}$
}

blood vessel development

cell cycle

nucleotide-binding

ubl conjugation

positive regulation of cell communication

intracellular organelle lumen

transcription from RNA polymerase II promoter

limb development

Stem cell maintenance

Golgi apparatus

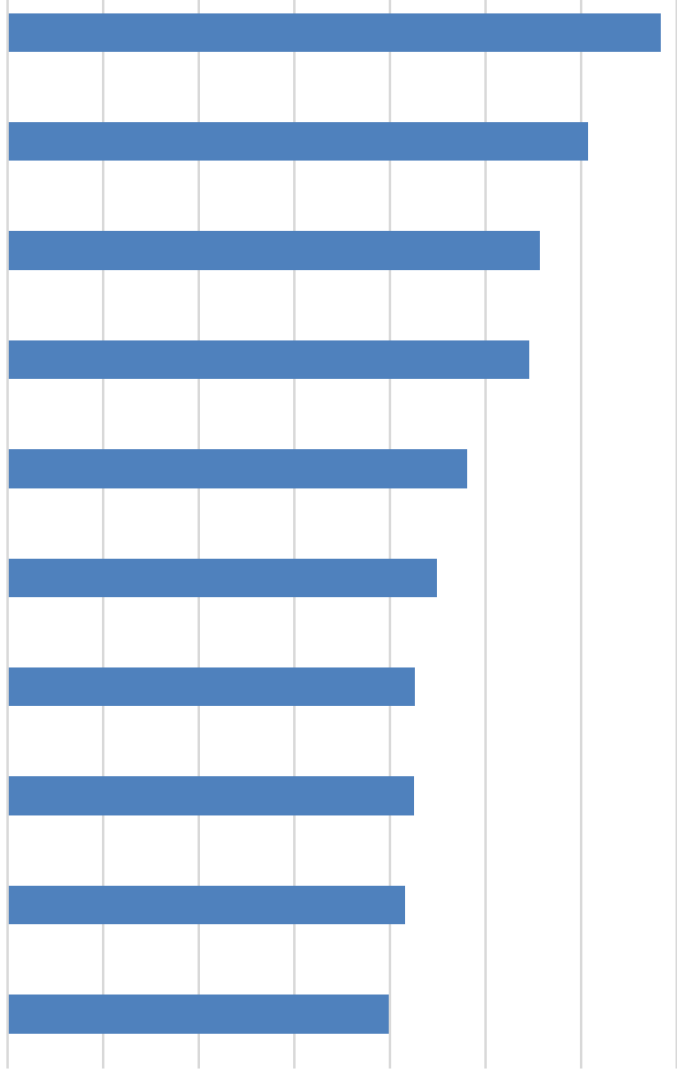

Fig 2. Functional Annotation Clustering analysis for known target genes of miRs-103/107. The known target gene list was exported from miRTarBase. The clustering analysis was performed by DAVID Functional Annotation Bioinformatics Resources v6.7. The rank is based on the Enrichment score, which represents mean $p$-value (in-log scale).

doi:10.1371/journal.pone.0134853.g002

that these differentially expressed genes were associated with a variety of processes (Fig 3). Consistent with our previous study [28], this assay suggested that miRs-103/107 played important roles in epithelial cell differentiation, proliferation, and regulation of cell-cell communication.

To determine the potential downstream regulatory system that impacts on miRs-103/107 function in stem cell maintenance, the GeneGo program was used to build a network including: (i) the differentially expressed genes in antagomir-103/107-treated HLEKs and (ii) stem-cellmaintenance-related direct target genes of miRs-103/107 (Fig 4). The largest network formed by Direct interactions algorithm contained: (i) four stem-cell maintenance-related direct target genes of miRs-103/107 (CREB5, KLF4, FOXO3A, and caveolin-1; S4 Table); and twenty-five differentially expressed genes (Fig 4). To validate this data, we chose nine differentially expressed genes in this network and confirmed their alterations by antagos-103/107 using qPCR analysis (Fig 5). Among these differentially expressed genes, c-Jun and JunD, components of the AP-1 complex [42, 43], were upregulated by antagos-103/107 treatment (Fig 5).

It has been reported that activation of AP-1 and its well-established upstream regulator p38 is required for epidermal keratinocyte differentiation [44-47]. Since miRs-103/107 promoted 


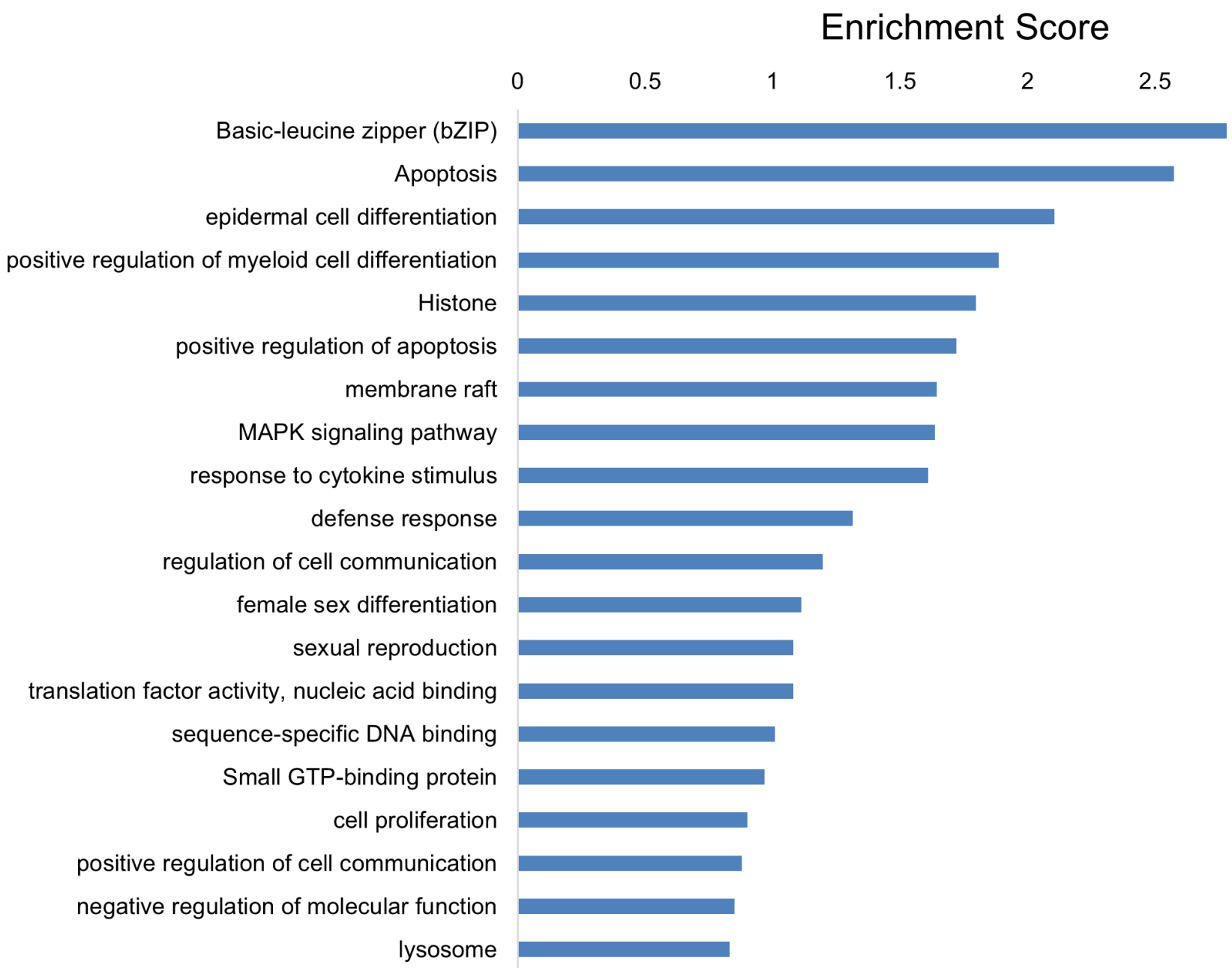

Fig 3. Functional Annotation Clustering analysis for differentially expressed genes in antago-103/107 treated HLEKs when compared with Irantago treated HLEKs. The clustering analysis was performed by DAVID Functional Annotation Bioinformatics Resources v6.7. The rank is based on the Enrichment score, which represents mean p-value (in-log scale).

doi:10.1371/journal.pone.0134853.g003 


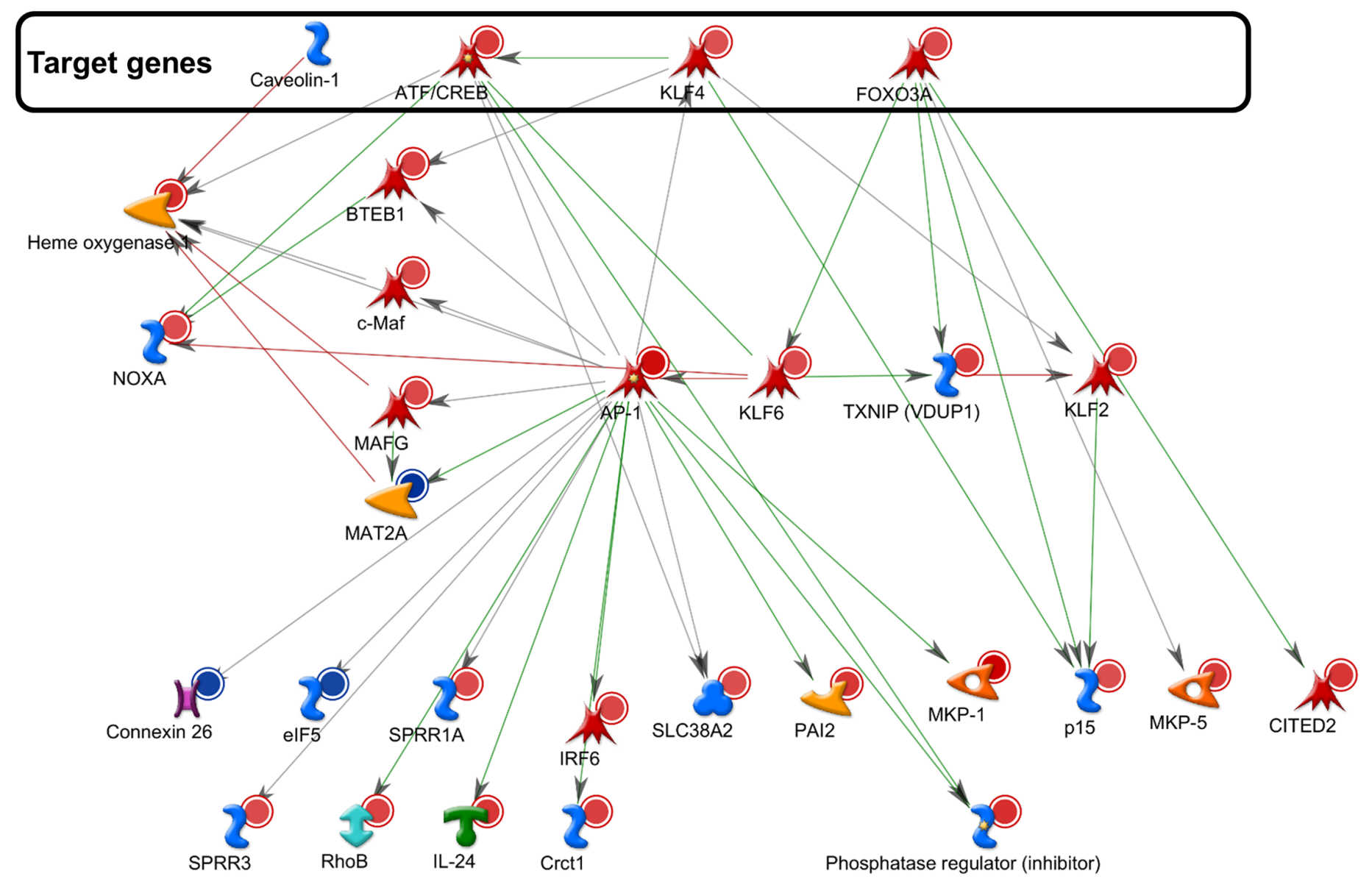

Fig 4. Networking analysis of miR-103/107-regulated genes. This network consists only of the seed nodes and of their direct interactions. Seed nodes included: (i) the differentially expressed genes in antagomir-103/107-treated HLEKs and (ii) miRs-103/107's direct target genes that involve in stem cell maintenance. The genes in the box are known direct targets of miRS-103/107.

doi:10.1371/journal.pone.0134853.g004

holoclone colonies [48]. Holoclones are the colonies formed by keratinocytes with great proliferative capacity, and such keratinocytes are considered to be "stem cells" [48]. HLEKs, treated with the p38 inhibitor SB203580 $(10 \mu \mathrm{M})$ for 3 days, gave rise to significantly more holoclones colonies compared with DMSO treated HLEKs (Fig 7). This indicates that inhibition of p38 enhances keratinocyte proliferative capacity. Since the miR-103/107 family contributes to a stem cell phenotype by increasing the proliferative capacity of keratinocytes [28], our findings strongly suggest that miRs-103/107 targeting of MAP3K7 leads to attenuation of the differentiation-related $\mathrm{p} 38$ pathway, and thus helps to maintain the proliferative capacity of keratinocytes (stem cells).

\section{Discussion}

We observed three different expression patterns over time among let7e, miR-199a-3p, miR328, miR-350, miR-99a, miR-199b*, miRs-103/107 and miR-342-3p, which are limbalpreferred microRNAs [28]. Expression of miR-328, miR-103 and miR-107 decreased at day 7 (Fig 1) when keratinocytes are activated and there is a marked increase in corneal epithelial proliferation [35]. This negative association between the expression of these three microRNAs and the proliferative rates of keratinocytes suggests that miR-328, miR-103 and miR-107 may 

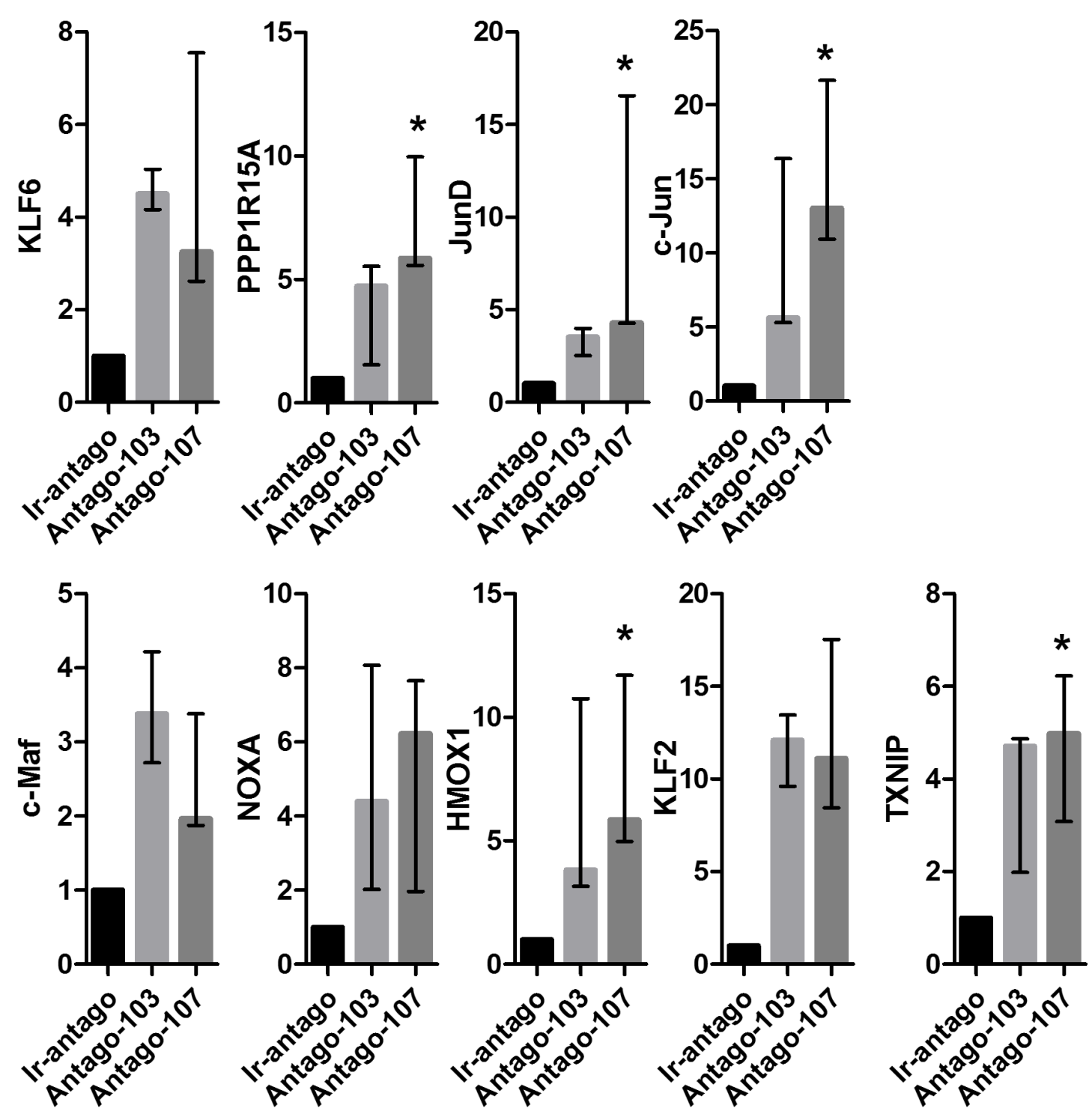

Fig 5. MiRs-103/107 negatively regulate AP-1 in HLEKs. Real time qPCR analysis of KLF6, PPP1R15A, JunD, c-Jun, c-Maf, NOXA, HMOX1, KLF2, and TXNIP levels in HLEKs that were treated with Ir-antago, Antago-103, or Antago-107. Values are median with range of four independent experiments. The significance of the differences between 3 groups was tested by non-parametric one-way ANOVA. ${ }^{*} p<0.05$.

doi:10.1371/journal.pone.0134853.g005

have an inhibitory effect on proliferation. In support of this idea, we have shown that miRs103/107 are negative regulators of cell cycle [28].

MiRs-103/107 increase the proliferative capacity of keratinocytes by targeting Wnt3a [28], suggesting a positive role of this microRNA family in stem cell maintenance. Inhibition of Wnt3a bypasses JNK-mediated repression of YAP1 [28], a determinant of epithelial stem cell proliferative capacity $[49,50]$, and promotes a stem cell phenotype [28]. In addition to YAP1, JNK also phosphorylates JunD and c-Jun [51], components of the AP-1 complex. Such phosphorylation increases activity of JunD and c-Jun [51]. Interestingly, inhibition of either JNK or the AP-1 complex represses keratinocyte differentiation [45-47]. Since depletion of miRs-103/ 107 activated JNK [28] and increased expression of JunD and c-Jun (Fig 5A), we believe that inhibition of the JNK/AP-1 pathways is another mechanism by which this microRNA family 


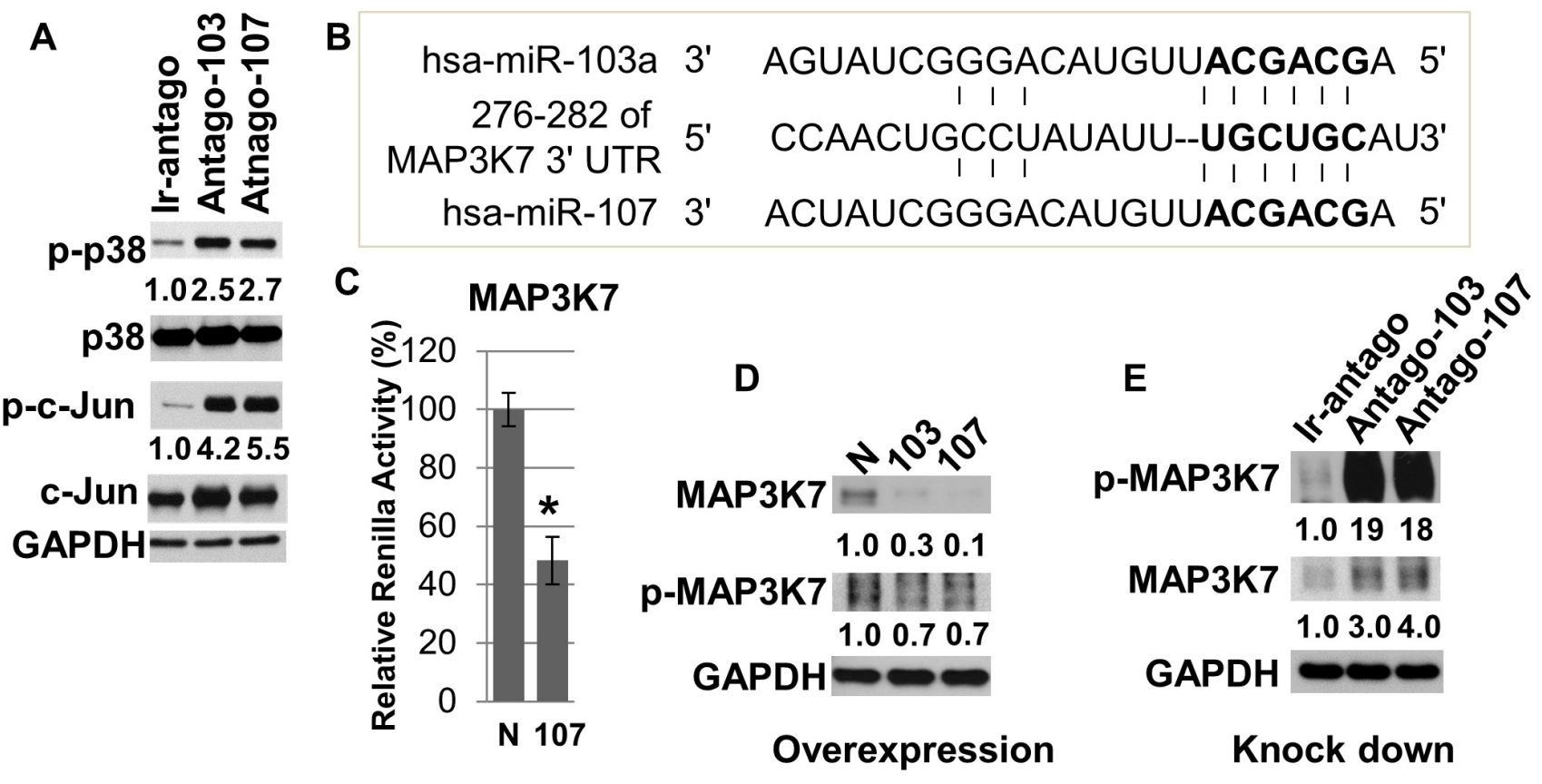

Fig 6. MiRs-103/107 negatively regulate p38/AP-1 pathway in HLEKs via directly targeting MAP3K7. (A) HLEKs were treated with Ir-antago, Antago103 or Antago-107 for 24 hours; then lysates were immunoblotted against phospho-p38 (p-p38), p38, phosphor-c-Jun (p-c-Jun), c-Jun and GAPDH. (B) Schematic diagrams of miR-103/107 binding sites in the 3'UTR region of MAP3K7 mRNA. Bold: seed sequences. (C) Screening the interaction of MAP3K7 with miR-103/107 using the psiCHECKTM-2 vector harboring 3' untranslated region (UTR) of MAP3K7. Constructs were co-transfected with either miRcontrol, or miR-107 into cells. Twenty four hour after transfection, firefly and renilla luciferase activities were measured using the Dual-Luciferase Reporter Assay System. N: precursor microRNA control, 107: precursor microRNA-107. T test was performed. ${ }^{*} p<0.05$. (D) Immunoblotting of endogenous total MAP3K7, phospho-MAP3K7 and GAPDH following over-expression of either pre-miR-negative control (N), pre-miR-103(103), or pre-miR-107(107). (E) HLEKs were treated with Ir-antago, Antago-103 or Antago-107 for 24 hours and lysates were immunoblotted for total MAP3K7, phospho-MAP3K7 and GAPDH. Numbers below the panels represent the normalized expression signal of proteins.

doi:10.1371/journal.pone.0134853.g006

DMSO

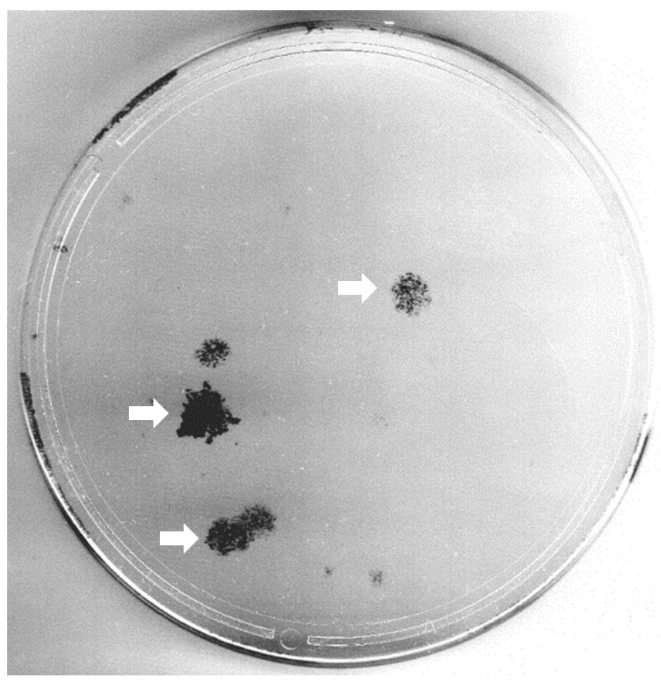

SB203580

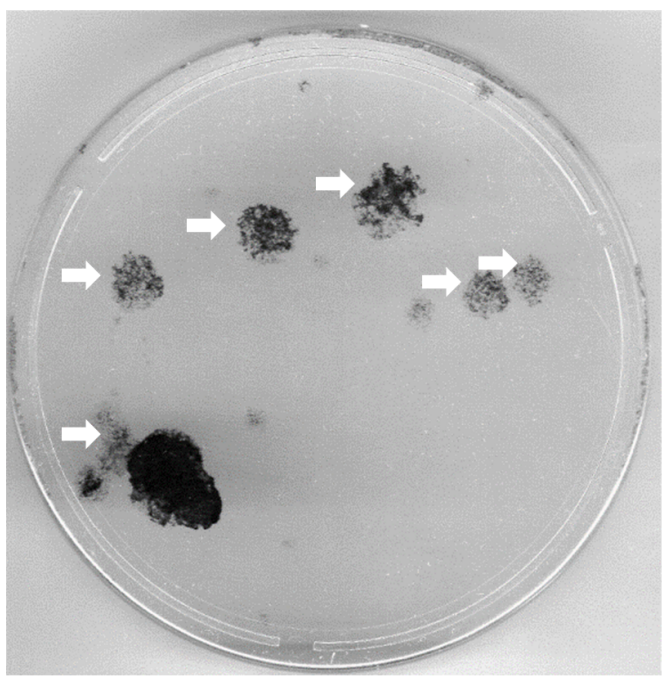

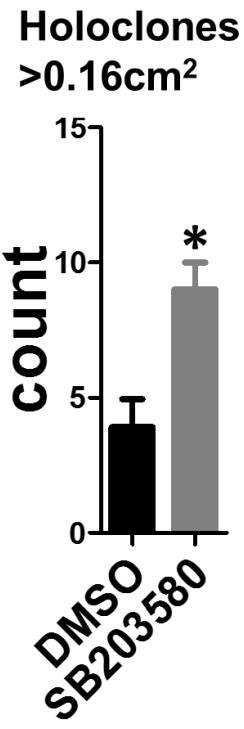

Fig 7. Inhibition of p38 increases holoclone formation. Representative holoclone forming assay of HLEKs following 3 day pre-treatment with SB203580 $(10 \mu \mathrm{M}$; p38 inhibitor) or DMSO. SB203580-treated keratinocytes gave rise to significantly more holoclones than DMSO. 200 cells per plate were seeded for each treatment. White arrow: holoclone $\left(\right.$ colonies $\left.>0.16 \mathrm{~cm}^{2}\right)$. T test was performed to calculate significance. ${ }^{*} \mathrm{p}<0.05$.

doi:10.1371/journal.pone.0134853.g007 
promotes an undifferentiated phenotype in LESCs. In addition to JNK, loss of miRs-103/107 also increased activity of p38, another MAPK (Fig 6A). Similar to JNK, p38 phosphorylates cJun and activates its DNA binding activity [52]. Interestingly, activation of the p38/AP-1 pathway is required for keratinocyte differentiation [44-47]. Taken together, our findings suggest that AP- 1 and the two MAPKs form a key regulatory network, by which miRs-103/107 may contribute to an undifferentiated state in LESCs. In support of this idea, c-Jun positively regulates expression of keratinocyte differentiation markers such as transglutaminase 1 [53, 54], loricrin [55, 56], and involucrin [54]. Moreover, JunD increases expression of Cystatin A [57], a gene specifically expressed in terminally differentiated keratinocytes [58].

Interestingly, MAP3K7, an upstream activator of MAPK/Jun pathway, is a direct target of miRs-103/107 (Fig 6B-6E). In addition to activating p38 and JNK, MAP3K7 also can activate IkappaB kinases, leading to the activation of NF-kappaB [59, 60]. Since NF-kappaB plays a role in limbal epithelial growth in vitro [61], it will be interesting to investigate whether NF-kappaB pathway contributes to the increased keratinocyte proliferative capacity by miRs-103/107.

Besides JunD and c-Jun, miRs-103/107 also affected other regulators of differentiation (Figs 4 and 5). For example, human Krüppel-like transcription factor 6 (KLF6) has been implicated in corneal physiology and integrity by positively regulating keratin12 [62], a keratin associated with corneal epithelial differentiation [5]. Embryonic stem cells lacking KLF6 failed to commit to a hematopoietic differentiation pathway and showed delayed expression of differentiation markers, Brachyury, Klf1 and Gata1 [63]. Furthermore, miRs-103/107 negatively regulated expression of c-Maf, which is a leucine zipper-containing transcription factor and is required for crystallin expression [64, 65], a marker of lens fiber cell differentiation [66]. Loss of c-Maf markedly impaired differentiation of lens fiber cells $[64,65]$, resulting in a severe developmental defect in lens. However, the role of c-Maf in limbal/corneal epithelia remains unknown [64].

\section{Conclusions}

In summary, much progress has been achieved in understanding how miRNAs function in a variety of tissues $[67,68]$ Since stem cells are a frequent site of neoplastic transformation [69-71], it is not surprising that much attention has been directed at understanding microRNAs in the context of cancer stem cells [72-77]. However, how microRNA regulate resting or "normal" stem cells, remains understudied. Our study begins to unravel the complexities underlying how a particular microRNA family influences limbal epithelial stem cell behavior as well as how this family is regulated.

\section{Supporting Information}

S1 Fig. Differential expression of miR-184 and miR-31 in limbal and corneal epithelia. MicroRNA qPCR analysis of miR-184 and miR-31 levels in corneal and limbal epithelia at postnatal day $3,7,14$, and 60 . Values are means \pm SD of three independent experiments. (TIF)

S2 Fig. Full western blot pictures with molecular weight standard. (TIF)

S1 Table. Primer sets for real time PCR.

(XLSX)

S2 Table. Genes that were upregulated by antago-103/107 treatment in HLEKs. (XLS) 
S3 Table. Genes that were downregulated by antago-103/107 treatment in HLEKs. (XLS)

S4 Table. Known direct target genes of miRs-103/107 that were associated with stem cell maintenance.

(XLSX)

\section{Acknowledgments}

Primary epidermal keratinocyte cultures and 3-D organotypic raft cultures were obtained from the Northwestern University Skin Disease Research Center (NU-SDRC) Skin Tissue Engineering Core facility; lentiviral constructs were obtained from the NU-SDRC DNA/RNA Delivery Core facility; and the NU-SDRC Morphology and Phenotyping Core facility assisted in morphological analysis. The NU Genomic Core assisted in the mRNA expression profiling studies. We thank Nihal Kaplan for useful discussions and critical reading of the manuscript.

\section{Author Contributions}

Conceived and designed the experiments: JKP RML HP. Performed the experiments: JKP WY JK RML HP. Analyzed the data: JKP WY JK RML HP. Wrote the paper: RML HP.

\section{References}

1. Lavker RM, Tseng SC, Sun TT. Corneal epithelial stem cells at the limbus: looking at some old problems from a new angle. Experimental eye research. 2004; 78(3):433-46. PMID: 15106923.

2. Peng $\mathrm{H}$, Kaplan N, Hamanaka RB, Katsnelson J, Blatt $\mathrm{H}$, Yang W, et al. microRNA-31/factor-inhibiting hypoxia-inducible factor 1 nexus regulates keratinocyte differentiation. Proceedings of the National Academy of Sciences of the United States of America. 2012; 109(35):14030-4. doi: 10.1073/pnas. 1111292109 PMID: 22891326; PubMed Central PMCID: PMC3435188.

3. Blanpain C, Fuchs E. Stem cell plasticity. Plasticity of epithelial stem cells in tissue regeneration. Science. 2014; 344(6189):1242281. doi: 10.1126/science.1242281 PMID: 24926024.

4. Cotsarelis G, Cheng SZ, Dong G, Sun TT, Lavker RM. Existence of slow-cycling limbal epithelial basal cells that can be preferentially stimulated to proliferate: implications on epithelial stem cells. Cell. 1989; 57(2):201-9. PMID: 2702690.

5. Schermer A, Galvin S, Sun TT. Differentiation-related expression of a major $64 \mathrm{~K}$ corneal keratin in vivo and in culture suggests limbal location of corneal epithelial stem cells. The Journal of cell biology. 1986; 103(1):49-62. Epub 1986/07/01. PMID: 2424919; PubMed Central PMCID: PMC2113783.

6. Amitai-Lange A, Altshuler A, Bubley J, Dbayat N, Tiosano B, Shalom-Feuerstein R. Lineage tracing of stem and progenitor cells of the murine corneal epithelium. Stem cells. 2015; 33(1):230-9. Epub 2014/ 09/05. doi: 10.1002/stem.1840 PMID: 25187087.

7. Buck RC. Ultrastructural characteristics associated with the anchoring of corneal epithelium in several classes of vertebrates. Journal of anatomy. 1983; 137 (Pt 4):743-56. PMID: 6668251; PubMed Central PMCID: PMC1171876

8. Collinson JM, Chanas SA, Hill RE, West JD. Corneal development, limbal stem cell function, and corneal epithelial cell migration in the Pax6(+/-) mouse. Investigative ophthalmology \& visual science. 2004; 45(4):1101-8. Epub 2004/03/24. PMID: 15037575.

9. Collinson JM, Morris L, Reid Al, Ramaesh T, Keighren MA, Flockhart JH, et al. Clonal analysis of patterns of growth, stem cell activity, and cell movement during the development and maintenance of the murine corneal epithelium. Developmental dynamics: an official publication of the American Association of Anatomists. 2002; 224(4):432-40. Epub 2002/08/31. doi: 10.1002/dvdy.10124 PMID: 12203735.

10. Thoft RA, Friend J. The $X, Y, Z$ hypothesis of corneal epithelial maintenance. Investigative ophthalmology \& visual science. 1983; 24(10):1442-3. PMID: 6618809.

11. Pellegrini G, Rama P, Matuska S, Lambiase A, Bonini S, Pocobelli A, et al. Biological parameters determining the clinical outcome of autologous cultures of limbal stem cells. Regenerative medicine. 2013; 8(5):553-67. Epub 2013/06/04. doi: 10.2217/rme.13.43 PMID: 23725042. 
12. Rama P, Matuska S, Paganoni G, Spinelli A, De Luca M, Pellegrini G. Limbal stem-cell therapy and long-term corneal regeneration. The New England journal of medicine. 2010; 363(2):147-55. Epub 2010/06/25. doi: 10.1056/NEJMoa0905955 PMID: 20573916.

13. Gangaraju VK, Lin H. MicroRNAs: key regulators of stem cells. Nat Rev Mol Cell Biol. 2009; 10(2): 116-25. Epub 2009/01/24. nrm2621 [pii] doi: 10.1038/nrm2621 PMID: 19165214.

14. Jackson SJ, Zhang Z, Feng D, Flagg M, O'Loughlin E, Wang D, et al. Rapid and widespread suppression of self-renewal by microRNA-203 during epidermal differentiation. Development. 2013; 140(9): 1882-91. Epub 2013/04/11. doi: 10.1242/dev.089649 PMID: 23571213; PubMed Central PMCID: PMC3631964.

15. Lena AM, Shalom-Feuerstein R, Rivetti di Val Cervo P, Aberdam D, Knight RA, Melino G, et al. miR-203 represses 'stemness' by repressing DeltaNp63. Cell death and differentiation. 2008; 15(7):1187-95. Epub 2008/05/17. doi: 10.1038/cdd.2008.69 PMID: 18483491.

16. Lewis BP, Burge CB, Bartel DP. Conserved seed pairing, often flanked by adenosines, indicates that thousands of human genes are microRNA targets. Cell. 2005; 120(1):15-20. doi: 10.1016/j.cell.2004. 12.035 PMID: 15652477.

17. Ning MS, Andl T. Custodians of the transcriptome: How microRNAs guard stemness in squamous epithelia. Stem cells. 2014. doi: 10.1002/stem.1922 PMID: 25524325.

18. Tiscornia G, Izpisua Belmonte JC. MicroRNAs in embryonic stem cell function and fate. Genes \& development. 2010; 24(24):2732-41. Epub 2010/12/17. doi: 10.1101/gad.1982910 PMID: 21159814; PubMed Central PMCID: PMC3003189.

19. Wang D, Zhang Z, O'Loughlin E, Wang L, Fan X, Lai EC, et al. MicroRNA-205 controls neonatal expansion of skin stem cells by modulating the PI(3)K pathway. Nature cell biology. 2013; 15(10):1153-63. doi: 10.1038/ncb2827 PMID: 23974039; PubMed Central PMCID: PMC3789848.

20. Xu N, Brodin P, Wei T, Meisgen F, Eidsmo L, Nagy N, et al. MiR-125b, a microRNA downregulated in psoriasis, modulates keratinocyte proliferation by targeting FGFR2. The Journal of investigative dermatology. 2011; 131(7):1521-9. doi: 10.1038/jid.2011.55 PMID: 21412257.

21. Yi R, Poy MN, Stoffel M, Fuchs E. A skin microRNA promotes differentiation by repressing 'stemness'. Nature. 2008; 452(7184):225-9. Epub 2008/03/04. doi: 10.1038/nature06642 PMID: 18311128.

22. Yu J, Ryan DG, Getsios S, Oliveira-Fernandes M, Fatima A, Lavker RM. MicroRNA-184 antagonizes microRNA-205 to maintain SHIP2 levels in epithelia. Proceedings of the National Academy of Sciences of the United States of America. 2008; 105(49):19300-5. Epub 2008/11/27. doi: 10.1073/pnas. 0803992105 PMID: 19033458; PubMed Central PMCID: PMC2587229.

23. Zhang L, Stokes N, Polak L, Fuchs E. Specific microRNAs are preferentially expressed by skin stem cells to balance self-renewal and early lineage commitment. Cell stem cell. 2011; 8(3):294-308. Epub 2011/03/03. doi: 10.1016/j.stem.2011.01.014 PMID: 21362569; PubMed Central PMCID: РМС3086714.

24. Lee SK, Teng $Y$, Wong HK, Ng TK, Huang L, Lei $P$, et al. MicroRNA-145 regulates human corneal epithelial differentiation. PloS one. 2011; 6(6):e21249. Epub 2011/06/28. doi: 10.1371/journal.pone. 0021249 PMID: 21701675; PubMed Central PMCID: PMC3119052.

25. Nakatsu MN, Ding Z, Ng MY, Truong TT, Yu F, Deng SX. Wnt/beta-catenin signaling regulates proliferation of human cornea epithelial stem/progenitor cells. Investigative ophthalmology \& visual science. 2011; 52(7):4734-41. Epub 2011/03/02. doi: 10.1167/iovs.10-6486 PMID: 21357396; PubMed Central PMCID: PMC3175950.

26. Pajoohesh-Ganji A, Stepp MA. In search of markers for the stem cells of the corneal epithelium. Biology of the cell / under the auspices of the European Cell Biology Organization. 2005; 97(4):265-76. Epub 2005/03/15. PMID: 15762848.

27. Puangsricharern V, Tseng SC. Cytologic evidence of corneal diseases with limbal stem cell deficiency. Ophthalmology. 1995; 102(10):1476-85. PMID: 9097795.

28. Peng H, Park JK, Katsnelson J, Kaplan N, Yang W, Getsios S, et al. microRNA-103/107 Family Regulates Multiple Epithelial Stem Cell Characteristics. Stem cells. 2015. doi: 10.1002/stem.1962 PMID: 25639731.

29. Peng H, Hamanaka RB, Katsnelson J, Hao LL, Yang W, Chandel NS, et al. MicroRNA-31 targets FIH-1 to positively regulate corneal epithelial glycogen metabolism. FASEB journal: official publication of the Federation of American Societies for Experimental Biology. 2012; 26(8):3140-7. doi: 10.1096/fj.11198515 PMID: 22532441; PubMed Central PMCID: PMC3405266.

30. Zhou M, Li XM, Lavker RM. Transcriptional profiling of enriched populations of stem cells versus transient amplifying cells. A comparison of limbal and corneal epithelial basal cells. The Journal of biological chemistry. 2006; 281(28):19600-9. Epub 2006/05/06. doi: 10.1074/jbc.M600777200 PMID: 16675456. 
31. Lagos-Quintana M, Rauhut R, Yalcin A, Meyer J, Lendeckel W, Tuschl T. Identification of tissue-specific microRNAs from mouse. Current biology: CB. 2002; 12(9):735-9. PMID: 12007417.

32. Yu J, Peng H, Ruan Q, Fatima A, Getsios S, Lavker RM. MicroRNA-205 promotes keratinocyte migration via the lipid phosphatase SHIP2. FASEB journal: official publication of the Federation of American Societies for Experimental Biology. 2010; 24(10):3950-9. doi: 10.1096/fj.10-157404 PMID: 20530248; PubMed Central PMCID: PMC2996908.

33. Liu CY, Zhu G, Westerhausen-Larson A, Converse R, Kao CW, Sun TT, et al. Cornea-specific expression of K12 keratin during mouse development. Current eye research. 1993; 12(11):963-74. Epub 1993/11/01. PMID: 7508359.

34. Thoft RA, Friend J. Biochemical transformation of regenerating ocular surface epithelium. Investigative ophthalmology \& visual science. 1977; 16(1):14-20. Epub 1977/01/01. PMID: 832961.

35. Tseng H, Matsuzaki K, Lavker RM. Basonuclin in murine corneal and lens epithelia correlates with cellular maturation and proliferative ability. Differentiation; research in biological diversity. 1999; 65(4): 221-7. Epub 2000/02/01. PMID: 10653358.

36. Wei ZG, Cotsarelis G, Sun TT, Lavker RM. Label-retaining cells are preferentially located in fornical epithelium: implications on conjunctival epithelial homeostasis. Investigative ophthalmology \& visual science. 1995; 36(1):236-46. Epub 1995/01/01. PMID: 7822151.

37. Ryan DG, Oliveira-Fernandes M, Lavker RM. MicroRNAs of the mammalian eye display distinct and overlapping tissue specificity. Molecular vision. 2006; 12:1175-84. PMID: 17102797.

38. Selbach M, Schwanhausser B, Thierfelder N, Fang Z, Khanin R, Rajewsky N. Widespread changes in protein synthesis induced by microRNAs. Nature. 2008; 455(7209):58-63. Epub 2008/08/01. doi: 10. 1038/nature07228 PMID: 18668040.

39. Baek D, Villen J, Shin C, Camargo FD, Gygi SP, Bartel DP. The impact of microRNAs on protein output. Nature. 2008; 455(7209):64-71. Epub 2008/08/01. doi: 10.1038/nature07242 PMID: 18668037; PubMed Central PMCID: PMC2745094.

40. Helwak A, Kudla G, Dudnakova T, Tollervey D. Mapping the human miRNA interactome by CLASH reveals frequent noncanonical binding. Cell. 2013; 153(3):654-65. Epub 2013/04/30. doi: 10.1016/j. cell.2013.03.043 PMID: 23622248; PubMed Central PMCID: PMC3650559.

41. Trajkovski M, Hausser J, Soutschek J, Bhat B, Akin A, Zavolan M, et al. MicroRNAs 103 and 107 regulate insulin sensitivity. Nature. 2011; 474(7353):649-53. Epub 2011/06/10. doi: 10.1038/nature10112 PMID: 21654750 .

42. Karin M, Liu Z, Zandi E. AP-1 function and regulation. Current opinion in cell biology. 1997; 9(2):240-6. PMID: 9069263.

43. Curran T, Franza BR Jr. Fos and Jun: the AP-1 connection. Cell. 1988; 55(3):395-7. PMID: 3141060.

44. Gandarillas A, Watt FM. Changes in expression of members of the fos and jun families and myc network during terminal differentiation of human keratinocytes. Oncogene. 1995; 11(7):1403-7. PMID: 7478564.

45. Connelly JT, Mishra A, Gautrot JE, Watt FM. Shape-induced terminal differentiation of human epidermal stem cells requires p38 and is regulated by histone acetylation. PloS one. 2011; 6(11):e27259. doi: 10.1371/journal.pone.0027259 PMID: 22073300; PubMed Central PMCID: PMC3206954.

46. Trappmann B, Gautrot JE, Connelly JT, Strange DG, Li Y, Oyen ML, et al. Extracellular-matrix tethering regulates stem-cell fate. Nature materials. 2012; 11(7):642-9. doi: 10.1038/nmat3339 PMID: 22635042.

47. Connelly JT, Gautrot JE, Trappmann B, Tan DW, Donati G, Huck WT, et al. Actin and serum response factor transduce physical cues from the microenvironment to regulate epidermal stem cell fate decisions. Nature cell biology. 2010; 12(7):711-8. doi: 10.1038/ncb2074 PMID: 20581838.

48. Barrandon $\mathrm{Y}$, Green $\mathrm{H}$. Three clonal types of keratinocyte with different capacities for multiplication. Proceedings of the National Academy of Sciences of the United States of America. 1987; 84(8): 2302-6. Epub 1987/04/01. PMID: 2436229; PubMed Central PMCID: PMC304638.

49. Schlegelmilch K, Mohseni M, Kirak O, Pruszak J, Rodriguez JR, Zhou D, et al. Yap1 acts downstream of alpha-catenin to control epidermal proliferation. Cell. 2011; 144(5):782-95. Epub 2011/03/08. doi: 10.1016/j.cell.2011.02.031 PMID: 21376238; PubMed Central PMCID: PMC3237196.

50. Zhang $\mathrm{H}$, Pasolli HA, Fuchs E. Yes-associated protein (YAP) transcriptional coactivator functions in balancing growth and differentiation in skin. Proceedings of the National Academy of Sciences of the United States of America. 2011; 108(6):2270-5. Epub 2011/01/26. doi: 10.1073/pnas.1019603108 PMID: 21262812; PubMed Central PMCID: PMC3038759.

51. Yazgan O, Pfarr CM. Regulation of two JunD isoforms by Jun $\mathrm{N}$-terminal kinases. The Journal of biological chemistry. 2002; 277(33):29710-8. doi: 10.1074/jbc.M204552200 PMID: 12052834. 
52. Humar M, Loop T, Schmidt R, Hoetzel A, Roesslein M, Andriopoulos N, et al. The mitogen-activated protein kinase $\mathrm{p} 38$ regulates activator protein 1 by direct phosphorylation of c-Jun. The international journal of biochemistry \& cell biology. 2007; 39(12):2278-88. doi: 10.1016/j.biocel.2007.06.013 PMID: 17689131.

53. Medvedev A, Saunders NA, Matsuura $\mathrm{H}$, Chistokhina A, Jetten AM. Regulation of the transglutaminase I gene. Identification of DNA elements involved in its transcriptional control in tracheobronchial epithelial cells. The Journal of biological chemistry. 1999; 274(6):3887-96. PMID: 9920944.

54. Rossi A, Jang SI, Ceci R, Steinert PM, Markova NG. Effect of AP1 transcription factors on the regulation of transcription in normal human epidermal keratinocytes. The Journal of investigative dermatology. 1998; 110(1):34-40. doi: 10.1046/j.1523-1747.1998.00071.x PMID: 9424084.

55. Jang SI, Steinert PM. Loricrin expression in cultured human keratinocytes is controlled by a complex interplay between transcription factors of the Sp1, CREB, AP1, and AP2 families. The Journal of biological chemistry. 2002; 277(44):42268-79. doi: 10.1074/jbc.M205593200 PMID: 12200429.

56. Ezhkova E, Pasolli HA, Parker JS, Stokes N, Su IH, Hannon G, et al. Ezh2 orchestrates gene expression for the stepwise differentiation of tissue-specific stem cells. Cell. 2009; 136(6):1122-35. doi: 10. 1016/j.cell.2008.12.043 PMID: 19303854; PubMed Central PMCID: PMC2716120.

57. Takahashi $H$, Asano K, Kinouchi M, Ishida-Yamamoto A, Wuepper KD, lizuka H. Structure and transcriptional regulation of the human cystatin A gene. The 12-O-tetradecanoylphorbol-13-acetate (TPA) responsive element-2 site (-272 to -278) on cystatin A gene is critical for TPA-dependent regulation. The Journal of biological chemistry. 1998; 273(28):17375-80. PMID: 9651321.

58. Steven AC, Steinert PM. Protein composition of cornified cell envelopes of epidermal keratinocytes. Journal of cell science. 1994; 107 (Pt 2):693-700. PMID: 8207091.

59. Ninomiya-Tsuji J, Kishimoto K, Hiyama A, Inoue J, Cao Z, Matsumoto K. The kinase TAK1 can activate the NIK-I kappaB as well as the MAP kinase cascade in the IL-1 signalling pathway. Nature. 1999; 398(6724):252-6. Epub 1999/03/27. PMID: 10094049.

60. Yamaguchi K, Shirakabe K, Shibuya H, Irie K, Oishi I, Ueno N, et al. Identification of a member of the MAPKKK family as a potential mediator of TGF-beta signal transduction. Science. 1995; 270(5244): 2008-11. Epub 1995/12/22. PMID: 8533096.

61. Cheng CY, Hsieh HL, Hsiao LD, Yang CM. PI3-K/Akt/JNK/NF-kappaB is essential for MMP-9 expression and outgrowth in human limbal epithelial cells on intact amniotic membrane. Stem cell research. 2012; 9(1):9-23. Epub 2012/03/31. doi: 10.1016/j.scr.2012.02.005 PMID: 22459175.

62. Chiambaretta F, Blanchon L, Rabier B, Kao WW, Liu JJ, Dastugue B, et al. Regulation of corneal keratin-12 gene expression by the human Kruppel-like transcription factor 6 . Investigative ophthalmology \& visual science. 2002; 43(11):3422-9. Epub 2002/10/31. PMID: 12407152.

63. Matsumoto N, Kubo A, Liu H, Akita K, Laub F, Ramirez F, et al. Developmental regulation of yolk sac hematopoiesis by Kruppel-like factor 6. Blood. 2006; 107(4):1357-65. Epub 2005/10/20. PMID: 16234353; PubMed Central PMCID: PMC1895396.

64. Kim JI, Li T, Ho IC, Grusby MJ, Glimcher LH. Requirement for the c-Maf transcription factor in crystallin gene regulation and lens development. Proceedings of the National Academy of Sciences of the United States of America. 1999; 96(7):3781-5. Epub 1999/03/31. PMID: 10097114; PubMed Central PMCID: PMC22371.

65. Kawauchi S, Takahashi S, Nakajima O, Ogino H, Morita M, Nishizawa M, et al. Regulation of lens fiber cell differentiation by transcription factor C-Maf. The Journal of biological chemistry. 1999; 274(27): 19254-60. Epub 1999/06/26. PMID: 10383433.

66. Vermorken AJ, Bloemendal $\mathrm{H}$. alpha-Crystallin polypeptides as markers of lens cell differentiation. Nature. 1978; 271(5647):779-81. PMID: 625352.

67. Wu H, Kong L, Zhou S, Cui W, Xu F, Luo M, et al. The role of microRNAs in diabetic nephropathy. Journal of diabetes research. 2014; 2014:920134. doi: 10.1155/2014/920134 PMID: 25258717; PubMed Central PMCID: PMC4165734.

68. Thum T, Condorelli G. Long Noncoding RNAs and MicroRNAs in Cardiovascular Pathophysiology. Circulation research. 2015; 116(4):751-62. doi: 10.1161/CIRCRESAHA.116.303549 PMID: 25677521.

69. Alison MR. Liver stem cells: implications for hepatocarcinogenesis. Stem cell reviews. 2005; 1(3): 253-60. PMID: 17142862.

70. Boehnke K, Falkowska-Hansen B, Stark HJ, Boukamp P. Stem cells of the human epidermis and their niche: composition and function in epidermal regeneration and carcinogenesis. Carcinogenesis. 2012; 33(7):1247-58. doi: 10.1093/carcin/bgs136 PMID: 22461521.

71. Thieu K, Ruiz ME, Owens DM. Cells of origin and tumor-initiating cells for nonmelanoma skin cancers. Cancer letters. 2013; 338(1):82-8. doi: 10.1016/j.canlet.2012.05.008 PMID: 22579650; PubMed Central PMCID: PMC3422447. 
72. Zhao B, Bian EB, Li J, Li J. New advances of microRNAs in glioma stem cells, with special emphasis on aberrant methylation of microRNAs. Journal of cellular physiology. 2014; 229(9):1141-7. doi: 10.1002/ jcp.24540 PMID: 24374932.

73. Qi W, Liang W, Jiang H, Miuyee Waye $M$. The function of miRNA in hepatic cancer stem cell. BioMed research international. 2013; 2013:358902. doi: 10.1155/2013/358902 PMID: 24383051; PubMed Central PMCID: PMC3870627.

74. Chhabra R, Saini N. MicroRNAs in cancer stem cells: current status and future directions. Tumour biology: the journal of the International Society for Oncodevelopmental Biology and Medicine. 2014; 35(9): 8395-405. doi: 10.1007/s13277-014-2264-7 PMID: 24964962.

75. Takahashi RU, Miyazaki $\mathrm{H}$, Ochiya $\mathrm{T}$. The role of microRNAs in the regulation of cancer stem cells. Frontiers in genetics. 2014; 4:295. doi: 10.3389/fgene.2013.00295 PMID: 24427168; PubMed Central PMCID: PMC3879439.

76. Gao X, Jin W. The emerging role of tumor-suppressive microRNA-218 in targeting glioblastoma stemness. Cancer letters. 2014; 353(1):25-31. doi: 10.1016/j.canlet.2014.07.011 PMID: 25042866.

77. Khalaj M, Tavakkoli M, Stranahan AW, Park CY. Pathogenic microRNA's in myeloid malignancies. Frontiers in genetics. 2014; 5:361. doi: 10.3389/fgene.2014.00361 PMID: 25477897; PubMed Central PMCID: PMC4237136. 